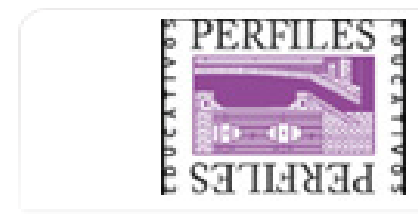

Perfiles Educativos

ISSN: 0185-2698

perfiles@unam.mx

Instituto de Investigaciones sobre la

Universidad y la Educación

México

González-Videgaray, MariCarmen; Hernández Zamora, Gregorio

Publicaciones de los investigadores educativos mexicanos en índices con liderazgo mundial

Perfiles Educativos, vol. XXXVI, núm. 144, 2014, pp. 31-49

Instituto de Investigaciones sobre la Universidad y la Educación

Distrito Federal, México

Disponible en: http://www.redalyc.org/articulo.oa? $\mathrm{id}=13230751003$

- Cómo citar el artículo

- Número completo

- Más información del artículo

- Página de la revista en redalyc.org

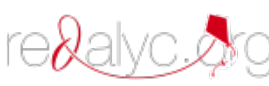

Sistema de Información Científica

Red de Revistas Científicas de América Latina, el Caribe, España y Portugal

Proyecto académico sin fines de lucro, desarrollado bajo la iniciativa de acceso abierto 


\title{
Publicaciones de los investigadores educativos mexicanos en índices con liderazgo mundial El caso de la UNAM
}

\author{
MariCarmen GonZÁlez-VidegaraY* | Gregorio Hernández Zamora**
}

En los últimos 20 años los programas de estímulos a la productividad académica impulsados en México han obligado a profesores e investigadores a publicar. Esta exigencia se relaciona con dos lógicas: una académica y otra política. Desde la académica, una investigación sólo se constituye como tal cuando es publicada en un medio apropiado. Desde la política, los mecanismos de compensación a salarios están condicionados al rendimiento académico y valoran publicaciones en medios con prestigio reconocido. En este trabajo analizamos los artículos sobre educación e investigación educativa publicados en revistas que participan en al menos uno de los dos índices internacionales más reconocidos (Journal Citation Reports y Scopus), por parte de investigadores adscritos a la Universidad Nacional Autónoma de México. Los resultados podrían interpretarse como un indicador de que la productividad académica es baja, sin embargo, consideramos que es necesario profundizar en este problema desde una perspectiva reflexiva y crítica.

Over the last twenty years, the incentive programs promoted in Mexico to boost academic productivity have obliged both teachers and researchers to publish. This requirement is related to two logics: one academic, the other political. From the academic perspective, research work is only considered as such once it has been published in an appropriate medium. From the political perspective, the economic stimulus mechanisms are conditional on academic performance and publication in prestigious journals. In this paper, the authors analyse articles on education and research published by researchers from the National Autonomous University of Mexico (UNAM by its Spanish acronym), in journals that are present in at least one of the two most-renowned international indexes: Journal Citation Reports and Scopus. The results may be interpreted as an indicator that the academic productivity level is low; however, the authors feel it is necessary to delve further into this problem from a reflexive, critical perspective.
Palabras clave

Arbitraje científico

Relación investigación-

docencia

Política universitaria

Publicaciones científicas

Rendimiento académico

Sistemas de evaluación

Trabajo docente

Keywords

Scientific peer review

Research-teaching relationship

University policy

Scientific publications

Academic performance

Evaluation systems

Teaching work

Recepción: 14 de abril de 2013 | Aceptación: 31 de mayo de 2013

* Doctora en Ingeniería Industrial, Maestra en Educación, actuaria. Profesora titular "C" tiempo completo en la Facultad de Estudios Superiores Acatlán de la Universidad Nacional Autónoma de México. Investigadora educativa en el área de tecnologías de información y comunicación, y matemáticas. Publicaciones recientes: (2011, en coautoría con J.H. del Río Martínez), Ambientes virtuales y objetos de aprendizaje, Naucalpan, Universidad Nacional Autónoma de México-Facultad de Estudios Superiores Acatlán; (2009, en coautoría con G. Hernández Zamora y J.H. del Río Martínez), "Learning Objects in Theory and Practice: A vision from Mexican university teachers", Computers \& Education: An International Journal, vol. 53, núm. 4, pp. 1330-38. CE: mcgv@unam.mx

** Doctor en Lengua y Cultura Escrita, Maestro en Ciencias, sociólogo. Profesor-investigador visitante, titular "C", en la Universidad Autónoma Metropolitana Cuajimalpa. Miembro del SNI. Especialista en el área de cultura escrita y educación. Publicaciones recientes: (2013), "Cultura escrita en espacios no escolares", en A. Carrasco y G. López-Bonilla (coords.), Lenguaje y educación: temas de investigación educativa en México, México, Fundación SM/ Consejo Puebla de Lectura, pp. 239-286; (2010), Decolonizing Literacy: Mexican lives in the era of global capitalism, Bristol, Multilingual Matters. CE: ghernandez@correo.cua.uam.mx 


\section{INTRODUCCIÓN ${ }^{1}$}

En los últimos 20 años, los programas de estímulos a la productividad académica impulsados en México (Rueda et al., 2010) han obligado a quienes somos profesores o investigadores a publicar. Lo mismo ocurre en la Universidad Nacional Autónoma de México (UNAM) que en otras universidades. Esta exigencia se relaciona con dos tipos de lógicas: una académica y otra política.

Desde la lógica académica, como dice John Ziman (1968), una investigación sólo se constituye como tal cuando es publicada en un medio apropiado, en el cual es arbitrada por los pares académicos y revisada exhaustivamente por otros investigadores del mismo campo. De esta forma se construye conocimiento a través de un proceso social colectivo y riguroso, se participa en el diálogo mundial sobre aspectos relevantes, y se fijan agendas de temas en los cuales conviene invertir los mayores esfuerzos.

Por otro lado, desde la lógica política, los programas de estímulo al rendimiento académico son justificados como parte de las políticas de "modernización" y "excelencia" (Sánchez Dromundo, 2005) ligadas y subordinadas, a su vez, a una "economía darwinista" que opone las ideas de competitividad y productividad a las de colaboración y solidaridad. Así, el ingreso a sistemas de reconocimiento y estímulos, es decir, los mecanismos de compensación a salarios sistemáticamente deteriorados, están condicionados al llamado rendimiento académico y valoran, es decir, otorgan puntos, sobre todo a publicaciones en "medios con prestigio reconocido” (UNAM-DGAPA, 1996).

Además, los programas de estímulo y sistemas de reconocimiento exigen que las publicaciones sean citadas por otros investigadores, también en medios prestigiados. Las citas se han convertido en un instrumento cuantitativo, que pretende ser objetivo, para medir el impacto de nuestros artículos de investigación. Por decirlo así, un artículo de investigación es "valioso" si es leído y comentado por otros investigadores del mismo campo, aunque en algunos casos las citas puedan ser críticas o ataques al artículo original.

La probabilidad de que uno de nuestros artículos sea citado depende efectivamente de la calidad de su aportación, pero también está en función de la visibilidad de la revista en la que apareció. Es decir, una revista que es leída por más investigadores tiene mayor oportunidad de que sus artículos sean citados. En general, las revistas en idioma inglés suelen tener más lectores y, por lo tanto, podrían recibir más citas en ése o en otros idiomas. Sin embargo, también existe una cantidad mayor de revistas en inglés, lo cual multiplica el número de artículos producidos y, por lo tanto, hace también menos probable que un artículo en particular sea leído.

Existen otros factores que afectan la probabilidad de que un artículo sea citado. Algunas revistas están catalogadas en ciertos índices o motores de búsqueda. Los índices de mayor prestigio internacional como el Journal Citation Reports (Thomson Reuters, 2012) o Scopus (Elsevier, 2013), son consultados por gran parte de los investigadores mundiales. Así, si publicamos en una revista indizada en ellos, tenemos la oportunidad de que estos investigadores vean nuestro artículo. Pero no sólo eso. Estos servicios brindan herramientas de búsqueda poderosas, como localizar artículos por título, tópico, palabras clave, adscripción del autor, nombre de la revista, entre otras. Además, permiten refinar las búsquedas por distintas categorías y brindan estadísticas sobre las publicaciones. Por lo tanto, las revistas que pertenecen a estos índices incrementan su visibilidad y su oportunidad de citas.

\footnotetext{
1 Esta investigación ha sido posible gracias al apoyo del Programa de Apoyo a Proyectos de Investigación para el Mejoramiento de la Enseñanza PAPIME PE 300 713, patrocinado por la Dirección General de Asuntos del Personal Académico de la UNAM.
} 
Los índices no aceptan en sus catálogos a cualquier revista; por el contrario, tienen criterios de selección muy claros y rigurosos. Por ejemplo, el Journal Citation Reports o JCR (Testa, 2012) verifica el cumplimiento de cada serie periódica en cuanto a: fechas de publicación, convenciones editoriales, idioma, arbitraje riguroso, contenido editorial, diversidad internacional y análisis de las citas. Así, este índice pretende englobar las mejores revistas especializadas del mundo, o si se quiere, las publicaciones de "prestigio reconocido". También se les denomina como "las grandes ligas" o la corriente principal (mainstream) de la investigación.

Además de lo anterior, el JCR califica la relevancia de las publicaciones a través del factor de impacto, desarrollado en 1955 por Eugene Garfield (2006; University of Pennsilvanya, 2011). El factor de impacto para un año dado se calcula como el número de citas recibidas durante los dos años anteriores por una revista, entre el total de artículos citables publicados en los mismos dos años. A partir de los estudios que realizó sobre este tema, Garfield fundó el llamado ISI (Institute for Scientific Information), que posteriormente fue adquirido por Thomson Reuters y hoy en día recibe el nombre de Web of Knowledge (2013). El Web of Knowledge es una plataforma digital que ofrece un grupo de servicios de búsqueda de información relacionada con la investigación en ciencias, ciencias sociales, humanidades y artes.

Tanto el JCR como el factor de impacto han sido muy criticados (Seglen, 1997), pues se considera que su funcionalidad es más apropiada para las revistas del área de las ciencias fácticas o "duras" que para las ciencias sociales y las humanidades, a las cuales pertenecería la investigación educativa. Se dice que este tipo de medidas favorece el "efecto San Mateo" (Merton, 1968), por el cual se beneficia a las revistas de los países más desarrollados y se afecta negativamente a las publicaciones e investigadores de las regiones más atrasadas. También puede preverse una interacción evidente: algunas revistas están indexadas en el JCR porque son visibles, y son más visibles precisamente porque están en el JCR.

Por estas razones han surgido alternativas para catalogar y calificar a las revistas. Entre estas iniciativas destacan: Scopus, EigenFactor, SciELO, DOAJ, Latindex y Google Académico. En ellas se amplía el rango de acceso y se consideran más revistas iberoamericanas. Por poner un ejemplo, si hacemos una búsqueda sencilla en la edición de Ciencias Sociales del JCR para 2011, encontramos en la categoría de Educación e Investigación Educativa (Education and Educational Research), 203 revistas. $^{2}$ Ahora, si hacemos una búsqueda similar en DOAJ, que es un directorio de revistas de acceso abierto, se encuentran 711 revistas sobre el tema. En Scopus hay 493 revistas con la palabra "education" en el título y otras cuatro con las palabras: educación, educativo o educativa. ${ }^{3}$

Sin embargo, el JCR sigue siendo líder en muchos sentidos porque provee de herramientas para cuantificar la investigación. Permite hacer mapas de citas, calificar el factor de impacto de las revistas y la influencia de los artículos, y cuenta con bases de datos a las que se tiene acceso con facilidad, si se paga el servicio, como ocurre en la UNAM. Además, las citas referenciadas en JCR parecen ser las de más valor para los criterios manejados por los programas de rendimiento académico, el Sistema Nacional de Investigadores o los índices internacionales de desarrollo del Banco Mundial (The World Bank, 2012). Tal vez por estas razones se insiste en la necesidad de que los investigadores publiquen en revistas indizadas en este sitio.

A pesar de lo anterior, Weiss y Gutiérrez (2003) han señalado que la producción de

2 Por supuesto, independientemente de ellas puede haber artículos relacionados con la educación y publicados en otras revistas.

3 Esta revisión no es exhaustiva, puesto que existen revistas especializadas en educación que no incluyen estas palabras en su título. 
los investigadores educativos mexicanos en revistas internacionales extranjeras es reducida. El estudio realizado por estos autores se aboca mayoritariamente a describir las publicaciones mexicanas y describe los datos correspondientes a los años entre 1993 y 2001. En la actualidad el Consejo Mexicano para la Investigación Educativa (COMIE) trabaja en el documento que reseña lo acontecido en los siguientes diez años.

Conviene pues preguntarse cuál es la participación real de los investigadores educativos mexicanos en la llamada corriente principal de las publicaciones mundiales. En particular, en este trabajo analizamos los artículos sobre educación e investigación educativa, publicados en revistas que participan en al menos uno de los dos índices internacionales más reconocidos (JCR y Scopus), por parte de investigadores adscritos a la UNAM. Si bien se trata de un estudio parcial, consideramos que brinda un panorama interesante para los investigadores de Iberoamérica.

A partir de los resultados obtenidos, se hace un análisis crítico y reflexivo acerca de la efectividad de los actuales programas de estímulo y reconocimiento académico, tanto para producir artículos de investigación de alto impacto, como para promover la mejora de la educación en nuestro país. Asimismo, se cuestiona la forma en que actualmente se miden el rendimiento y la productividad académica.

\section{MÉTODo}

Con el fin de delinear el panorama de la participación de los investigadores educativos de la UNAM en los índices de publicaciones líderes mundiales, se efectuaron las siguientes consultas:

a) Para ponderar la participación de las publicaciones mexicanas en los índices mundiales, se identificaron las revistas del área de Educación en el Índice de
Revistas Mexicanas de Investigación Científica y Tecnológica del CONACyT, así como los índices internacionales a los cuales pertenece cada una de ellas.

b) Con el mismo objetivo anterior, en el índice JCR 2011 Social Sciences Edition, del Web of Knowledge, se obtuvo el número total de revistas en la categoría Education and Educational Research. En este grupo se identificaron las revistas en español y una revista mexicana. No se efectuó este análisis en Scopus, ya que su clasificación sólo se refiere a ciencias sociales y no delimita las revistas de educación.

c) Con el fin de enmarcar este artículo dentro de la producción de investigación educativa mundial y contar con un medio de contraste, se identificaron las cinco revistas con mayor factor de impacto dentro del JCR 2011, así como su respectivo factor de impacto. Se ubicaron las instrucciones para los autores que pretendan colaborar en ellas y se elaboró un cuadro para comparar esta información con la correspondiente de las revistas indexadas en CONACyT.

d) Para brindar una idea acerca de la proporción de investigadores educativos en México, se tomaron los datos del número de investigadores que han formado parte del Sistema Nacional de Investigadores (SNI) del CONACyT, por año, y por campo de la ciencia.

e) Para observar la producción de investigación educativa por parte de la UNAM, en Current Contents Connect (CCC) del Web of Knowledge, se efectuó la búsqueda de artículos publicados por académicos adscritos a esta institución. El perfil de búsqueda y el número de resultados se muestran en la Fig. 1. Se filtraron únicamente artículos originales de investigación y artículos de revisión. Posteriormente 
el total de artículos se refinó para obtener sólo los que corresponden a Education and Educational Research.
Se efectuaron estadísticas descriptivas de los datos obtenidos, con la propia base de datos.

Figura 1. Perfil de búsqueda en Current Contents Connect

\begin{tabular}{|c|c|c|}
\hline Set & Results & Save History / Create Alert \\
\hline$\# 4$ & 18 & $\begin{array}{l}\text { \#2 AND } \# 1 \\
\text { Refined by: Research Areas=( EDUCATION EDUCATIONLLL RESEARCH) } \\
\text { Databases=ABES, SBS, CM, LS, PCES, ECT, AH Timespan=All Years }\end{array}$ \\
\hline$\# 3$ & 31,393 & $\begin{array}{l}\text { \#2 AND \#1 } \\
\text { Databases=ABES, SBS, CM, LS, PCES, ECT, AH Timespan=AII Years }\end{array}$ \\
\hline$\# 2$ & 126,410 & $\begin{array}{l}(A D=(\text { mexico) }) \text { AND Document Types }=(\text { Article OR Review }) \\
\text { Databases }=A B E S, S B S, C M, L S, P C E S, E C T, A H \text { Timespan=AIr Years }\end{array}$ \\
\hline$\# 1$ & 31,806 & $\begin{array}{l}\text { (AD=("Univ Nacl Autonoma Mexico" OR unam OR "universidad nacional autonoma de mexico")) AND Document } \\
\text { Types=(Article OR Review) } \\
\text { Databases=ABES, SBS, CM, LS, PCES, ECT, AH Timespan=All Years }\end{array}$ \\
\hline
\end{tabular}

Fuente: elaboración propia.

f) Para continuar el análisis anterior, se realizó en la base de datos Scopus una búsqueda semejante a la del CCC, según se muestra en la Fig. 2. Se filtraron únicamente artículos originales de investigación y artículos de revisión. Puesto que la base no ofrece la categoría de educación o investigación educativa, se buscaron documentos de ciencias sociales con las palabras clave educat $^{*}$, teach ${ }^{*}$, learn ${ }^{*}$ o instruct ${ }^{*}$. Se efectuaron estadísticas descriptivas con los datos resultantes, con la propia base de datos.

Figura 2. Perfil de búsqueda en Scopus

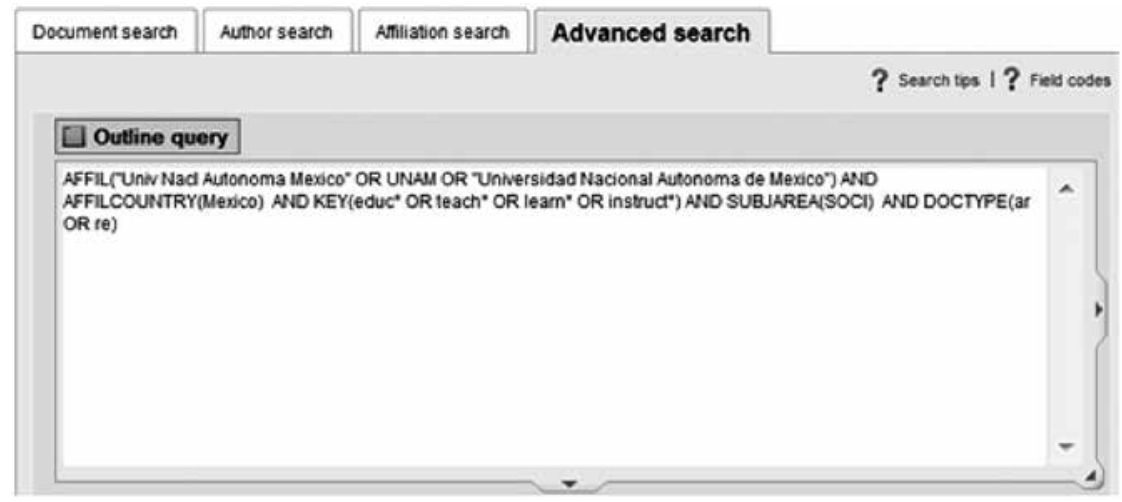

Fuente: elaboración propia.

\section{Resultados}

\section{Revistas en el índice del CONACyT}

El Cuadro 1 muestra las revistas especializadas de educación registradas por el CONACyT y algunos de los índices a los que éstas pertenecen.
El CONACyT no cataloga todas las revistas mexicanas; por ejemplo, no aparecen en esta lista Educación Matemática del Consejo Mexicano para la Investigación Educativa (COMIE), Didac, de la Universidad Iberoamericana, o Reencuentro, de la Universidad Autónoma Me- 
tropolitana-Xochimilco, entre muchas otras.

Sólo una revista mexicana de educación está indizada en JCR y dos en Scopus.

Cuadro 1. Índices a los que pertenecen las revistas especializadas mexicanas de humanidades y ciencias de la conducta/educación

\begin{tabular}{|c|c|c|c|c|c|c|}
\hline Revista & Institución & $\begin{array}{l}\text { Thomson } \\
\text { Reuters JCR }\end{array}$ & Scopus & DOAJ & SciELO & $\begin{array}{l}\text { Latindex } \\
\text { (UNAM) }\end{array}$ \\
\hline Educación Matemática & $\begin{array}{l}\text { Universidad } \\
\text { Pedagógica Nacional }\end{array}$ & & & & $\mathrm{x}$ & $\mathrm{x}$ \\
\hline Perfiles Educativos & UNAM & & $\mathrm{x}$ & $\mathrm{x}$ & $\mathrm{x}$ & $\mathrm{x}$ \\
\hline $\begin{array}{l}\text { Revista Mexicana de Investigación } \\
\text { Educativa }\end{array}$ & COMIE & & & $\mathrm{x}$ & $\mathrm{x}$ & $\mathrm{x}$ \\
\hline $\begin{array}{l}\text { REDIE. Revista Electrónica de } \\
\text { Investigación Educativa }\end{array}$ & $\begin{array}{l}\text { Universidad } \\
\text { Autónoma de Baja } \\
\text { California }\end{array}$ & & $\mathrm{x}$ & $\mathrm{x}$ & $\mathrm{x}$ & $\mathrm{x}$ \\
\hline $\begin{array}{l}\text { Revista Latinoamericana de } \\
\text { Investigación en Matemática Educativa }\end{array}$ & $\begin{array}{l}\text { Instituto Politécnico } \\
\text { Nacional }\end{array}$ & $\mathrm{x}$ & & $\mathrm{x}$ & $\mathrm{x}$ & $\mathrm{x}$ \\
\hline Revista de la Educación Superior & $\begin{array}{l}\text { Asociación Nacional } \\
\text { de Universidades } \\
\text { e Instituciones de } \\
\text { Educación Superior }\end{array}$ & & & $\mathrm{x}$ & $\mathrm{x}$ & $\mathrm{x}$ \\
\hline
\end{tabular}

Fuente: elaboración propia con datos del CONACyT, 2012.

Revistas de educación e investigación educativa indizadas en JCR

En el JCR edición Ciencias Sociales (Thomson Reuters, 2011) existen 203 revistas de educación e investigación educativa. Dentro de esta categoría, ocho revistas están en idioma español (Cuadro 2), aunque una de ellas es multilenguaje (Porta Linguarum). Todas son españolas, con excepción de la Revista Latinoamericana de Investigación en Matemática Educativa (RELIME), que es mexicana.

Cuadro 2. Revistas en idioma español indizadas en JCR 2011 Social Sciences Edition

\begin{tabular}{|c|c|c|}
\hline Revista & País & FI \\
\hline Comunicar (multilenguaje) & España & 0.470 \\
\hline Revista de Educación & España & 0.409 \\
\hline Revista Española de Pedagogía & España & 0.333 \\
\hline Cultura y Educación & España & 0.274 \\
\hline Enseñanza de las Ciencias & España & 0.188 \\
\hline Revista Latinoamericana de Investigación en Matemática Educativa-RELIME & México & 0.167 \\
\hline Educación XXI & España & 0.103 \\
\hline Porta Linguarum & España & 0.062 \\
\hline
\end{tabular}

Fuente: elaboración propia con datos mencionados.

$\mathrm{FI}=$ factor de impacto. 
El índice Scopus no ofrece la clasificación de las revistas por área de Educación e Investigación Educativa. Se encontraron 529 series periódicas cuyo título incluye los caracteres educat*, que son sólo un subconjunto de las revistas alrededor del tema educativo.

\section{Revistas con mayor factor}

\section{de impacto en JCR edición 2011}

De acuerdo con la consulta realizada en el JCR edición 2011 de Ciencias Sociales, dentro delárea de Educación e Investigación Educativa, el Cuadro 3 muestra cinco revistas especializadas con el mayor factor de impacto, así como la dirección electrónica donde se ubican las instrucciones para los autores que deseen colaborar en ellas. En el mismo cuadro se agregaron las seis revistas mexicanas indexadas por el CONACyT. Esto brinda una idea de cuáles son los temas líderes en la investigación educativa mundial.

Cuadro 3. Revistas de educación e investigación educativa en JCR 2011 Social Sciences Edition (las cinco primeras por FI) e indexadas por el CONACyT (las seis últimas)

\begin{tabular}{|c|c|c|c|}
\hline & Revista & FI & Instrucciones para los autores \\
\hline 1 & $\begin{array}{l}\text { Academy of Management } \\
\text { Learning \& Education }\end{array}$ & 4.800 & http://aom.org/Publications/AMLE/Information-for-Contributors.aspx \\
\hline 2 & Learning and Instruction & 3.732 & $\begin{array}{l}\text { http://www.elsevier.com/journals/ } \\
\text { learning-and-instruction/0959-4752/guide-for-authors }\end{array}$ \\
\hline 3 & $\begin{array}{l}\text { Review of Educational } \\
\text { Research }\end{array}$ & 3.169 & $\begin{array}{l}\text { http://www.sagepub.com/journals/Journal201854/ } \\
\text { manuscriptSubmission }\end{array}$ \\
\hline 4 & Educational Researcher & 2.931 & $\begin{array}{l}\text { http://www.sagepub.com/journals/Journal201856/ } \\
\text { manuscriptSubmission }\end{array}$ \\
\hline 5 & Reading Research Quarterly & 2.697 & $\begin{array}{l}\text { http://www.reading.org/General/Publications/index/writing_for_ } \\
\text { reading_research_quarterly.aspx }\end{array}$ \\
\hline 6 & Educación Matemática & - & http://www.revista-educacion-matematica.com/ \\
\hline 7 & Perfiles Educativos & - & http://www.iisue.unam.mx/perfiles/normas_editoriales.pdf \\
\hline 8 & $\begin{array}{l}\text { Revista Mexicana de } \\
\text { Investigación Educativa }\end{array}$ & - & $\begin{array}{l}\text { http://www.comie.org.mx/doc/rmie/documentos/nuevo_protocolo_ } \\
\text { para_colaboradores.pdf }\end{array}$ \\
\hline 9 & $\begin{array}{l}\text { REDIE. Revista Electrónica de } \\
\text { Investigación Educativa }\end{array}$ & - & http://redie.uabc.mx/enlaces/normas-editoriales-de-formato.html \\
\hline 10 & $\begin{array}{l}\text { Revista Latinoamericana de } \\
\text { Investigación en Matemática } \\
\text { Educativa }\end{array}$ & 0.167 & http://www.clame.org.mx/relime.htm \\
\hline 11 & $\begin{array}{l}\text { Revista de la Educación } \\
\text { Superior }\end{array}$ & - & http://publicaciones.anuies.mx/le-interesa-publicar-en-anuies \\
\hline
\end{tabular}

A continuación, el Cuadro 4 detalla algunas de las normas que las revistas anteriores solicitan cumplir a los autores que deseen publicar en ellas. En todos los casos se trata de revistas arbitradas y el cumplimiento de las normas no garantiza (aunque sí hace posible) la aceptación del manuscrito. En la columna seis, las siglas APA significan: American Psychological Association (Asociación
Psicológica Americana). En la columna siete, la estructura IMRD corresponde, por sus siglas, a: Introducción, Material y método, Resultados y Discusión; es la estructura típica de los artículos de investigación original, al menos dentro de las ciencias fácticas. Puede observarse que esta estructura no es indispensable en el ámbito de la investigación educativa. 


\section{Cuadro 4. Algunas normas solicitadas por las revistas para publicar artículos de investigación original}

\begin{tabular}{ccclclcc}
\hline Revista & $\begin{array}{c}\text { Ocultar } \\
\text { identidad } \\
\text { de autor(es) }\end{array}$ & $\begin{array}{c}\text { Extensión } \\
\text { mínima }\end{array}$ & $\begin{array}{c}\text { Extensión } \\
\text { máxima }\end{array}$ & $\begin{array}{c}\text { Buen } \\
\text { inglés }\end{array}$ & $\begin{array}{c}\text { Formato para } \\
\text { el aparato } \\
\text { crítico }\end{array}$ & $\begin{array}{c}\text { Estructura } \\
\text { IMRD }\end{array}$ & $\begin{array}{c}\text { Formato } \\
\text { para cuadros } \\
\text { y figuras }\end{array}$ \\
\hline 1 & Sí & 20 páginas & 40 páginas & Sí & APA 6th & No & Sí \\
\hline 2 & Sí & 4,500 palabras & 8,000 palabras & Sí & APA 6th & Sí & Sí \\
\hline 3 & Sí & No & 15,000 palabras & No & APA 6th & No & Sí \\
\hline 4 & Sí & No & 5,000 palabras & Sí & APA 6th & No & Sí \\
\hline 5 & Sí & No & No & No & APA 6th & No & No \\
\hline 6 & Sí & No & 10,000 palabras & No & APA & No & Sí \\
\hline 7 & No & 7,000 palabras & 10,500 palabras & No & Formato propio & No & Sí \\
\hline 8 & Sí & 6,000 palabras & 9,000 palabras & No & Harvard & No & No \\
\hline 9 & No & 15 cuartillas & 20 cuartillas & No & APA & No & Sí \\
\hline 10 & Sí & No & 9,000 palabras & No & APA 4th & $\begin{array}{c}\text { Sí } \\
\text { (modificada) }\end{array}$ & Sí \\
\hline 11 & No & No & 9,000 palabras & & APA & No & Sí \\
\hline
\end{tabular}

Fuente: elaboración propia con datos mencionados.

Número de investigadores nacionales por campo de la Ciencia en SNI

A partir del Sistema Integrado de Información sobre Información Científica y Tecnológica (SIICyT) del CONACyT, se presenta en la Fig. 3 el porcentaje correspondiente a Pedagogía, tan sólo como un acercamiento al dato del número de investigadores educativos en México. El número de investigadores en Pedagogía es de 281 contra, por ejemplo, 2 mil 882 de Ciencias de la vida.

Figura 3. Proporción de investigadores del SNI por campo de la ciencia.

La proporción destacada corresponde a Pedagogía
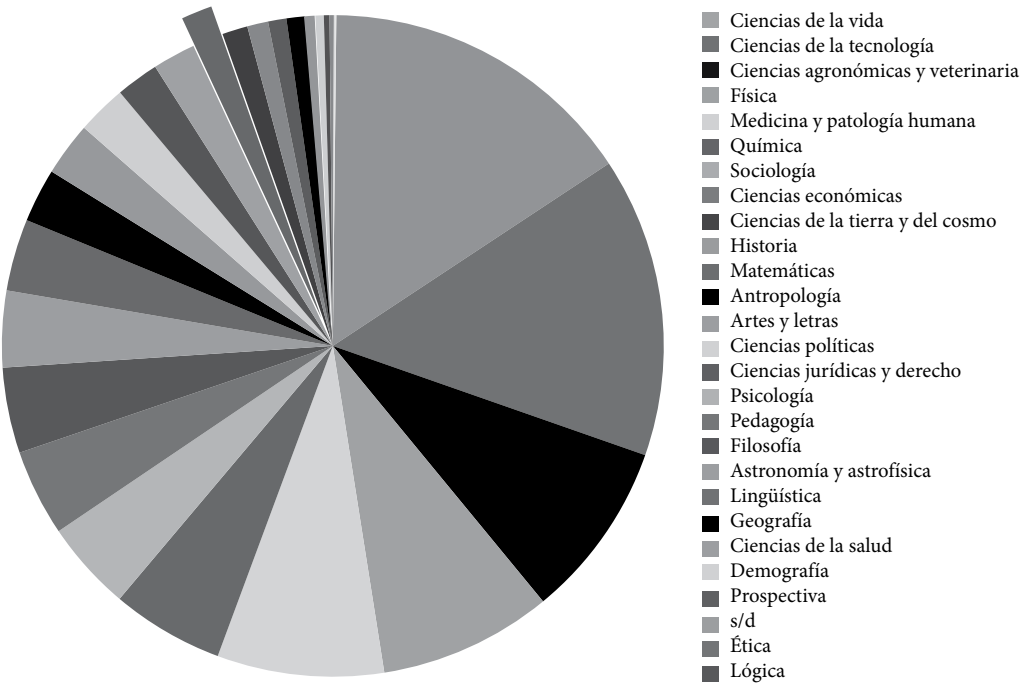

Fuente: CONACyT, 2013. 
Publicaciones en CCC de investigadores adscritos a la UNAM

Según la búsqueda en el servicio CCC, la UNAM ha publicado 31 mil 393 artículos de investigación en todas las áreas del conocimiento. Tan solo 18 de ellos ( 0.06 por ciento) se circunscriben al área de Educación e Investigación
Educativa. La mayoría (17) son artículos de investigación originales y uno es de revisión. Todos los artículos están en inglés.

La Fig. 4 muestra los títulos de las revistas en las cuales han publicado académicos de la UNAM. Destacan las áreas de Ciencias, Medicina, Química y Tecnología en educación.

Figura 4. Artículos de educación e investigación educativa publicados por académicos de la UNAM e indizados en Current Contents Connect por revista, ordenados por número de registros

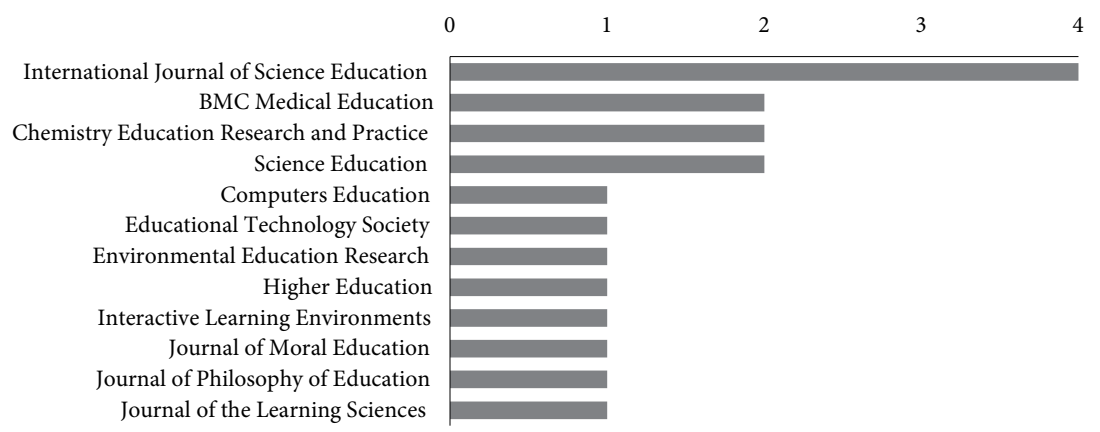

Fuente: elaboración propia con los datos mencionados.

En el Cuadro 5 se indica la procedencia de los autores de los mismos artículos. Si bien el número de datos es escaso, parece desprenderse del cuadro que el mayor número de publicaciones corresponde a investigadores de Química. También parecería que hay más producción por parte de las ciencias llamadas "duras", aún dentro del área de investigación educativa.

Cuadro 5. Número de artículos de investigación original de educación e investigación educativa publicados por académicos de la UNAM e indizados en Current Contents Connect por adscripción del autor(es).

\begin{tabular}{lc}
\hline \multicolumn{1}{c}{ Dependencia } & Número de artículos \\
\hline Facultad de Química & 5 \\
\hline Centro de Instrumentos $^{*}$ & 3 \\
\hline Facultad de Medicina & 2 \\
\hline Centro de Ciencias Aplicadas y Desarrollo Tecnológico & 1 \\
\hline Instituto de Investigaciones sobre la Universidad y la Educación & 1 \\
\hline Centro de Investigación en Ecosistemas, Morelia & 1 \\
\hline Facultad de Estudios Superiores Acatlán & 1 \\
\hline Instituto de Investigaciones Económicas & 1 \\
\hline Sin especificar & 3 \\
\hline TOTAL & 18 \\
\hline
\end{tabular}

${ }^{*}$ Un artículo con coautor del Colegio de Ciencias y Humanidades.

Fuente: elaboración propia. 
Por su parte, la Fig. 5 muestra el total de artículos de investigación original anteriores, organizados por año de publicación. No parece haber una tendencia creciente en estos datos.

Figura 5. Número de artículos de investigación original de educación e investigación educativa publicados por académicos de la UNAM e indizados en Current Contents Connect por año

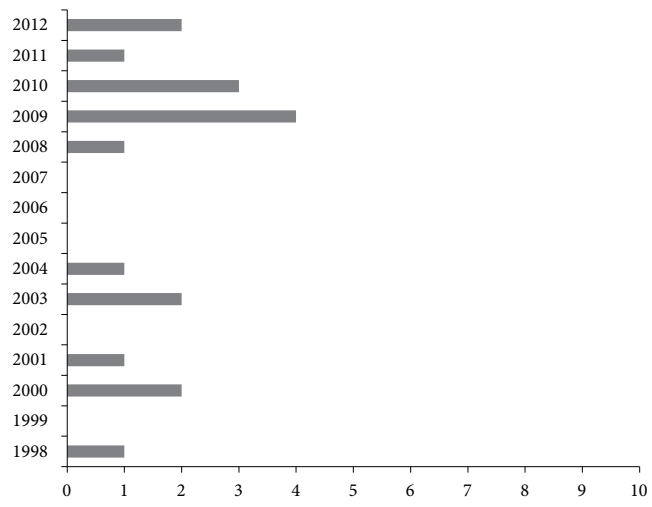

Fuente: elaboración propia.
Si bien los autores adscritos a la UNAM son mexicanos, la Fig. 6 muestra que hay colaboración con otros países.

Figura 6. País de origen de autores y coautores

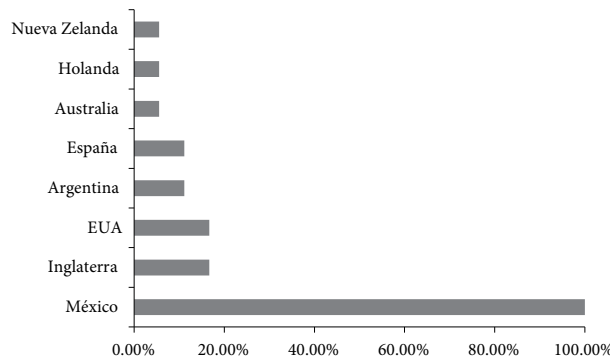

Fuente: elaboración propia con los datos mencionados.

La Fig. 7 brinda un panorama de la colaboración interinstitucional que puede verse en los diferentes artículos publicados.

Figura 7. Institución de adscripción de autores y coautores

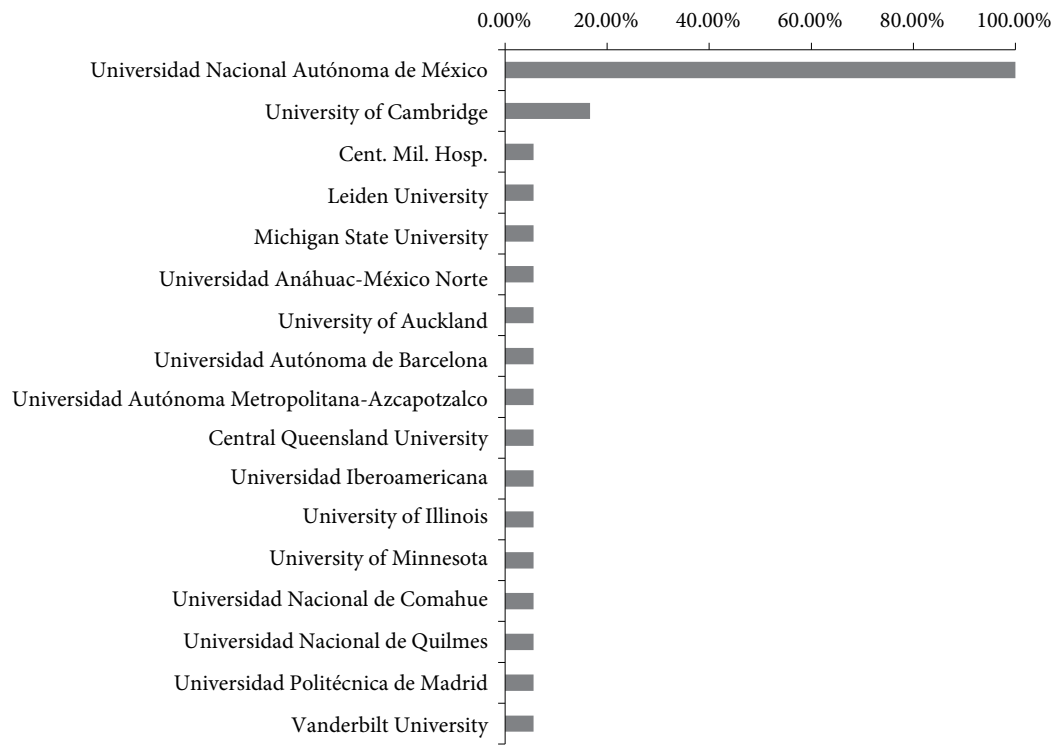

Fuente: elaboración propia con los datos mencionados. 


\section{Publicaciones en Scopus}

de investigadores adscritos a la UNAM

Se realizó una búsqueda similar en el catálogo de Scopus, que contempla un número mayor de revistas. El total de artículos de investigación originales publicados por académicos de la UNAM y registrados en Scopus fue de 40 mil 158. De ellos se extrajeron 107 sobre educación, es decir, 0.27 por ciento, de los cuales 101 son artículos de investigación original y seis son de revisión.

La Fig. 8 muestra las trece revistas indexadas en Scopus con más artículos sobre educación publicados por académicos de la UNAM. Sobresalen, además de Perfiles Educativos, las áreas de Química y Medicina.

Figura 8. Número de artículos de investigación sobre educación publicados por académicos de la UNAM, por revista indizada en Scopus (sólo las trece primeras)

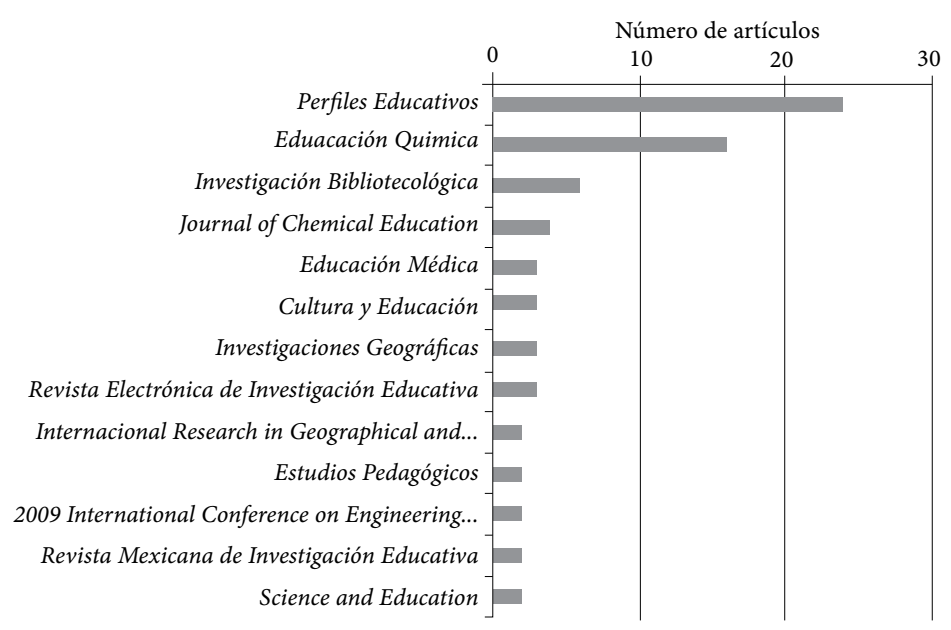

Fuente: elaboración propia.

La Fig. 9 muestra el número de artículos publicados por año. Se advierte una tendencia creciente moderada.

Figura 9. Número de artículos sobre educación publicados por académicos de la UNAM por año e indizados en Scopus

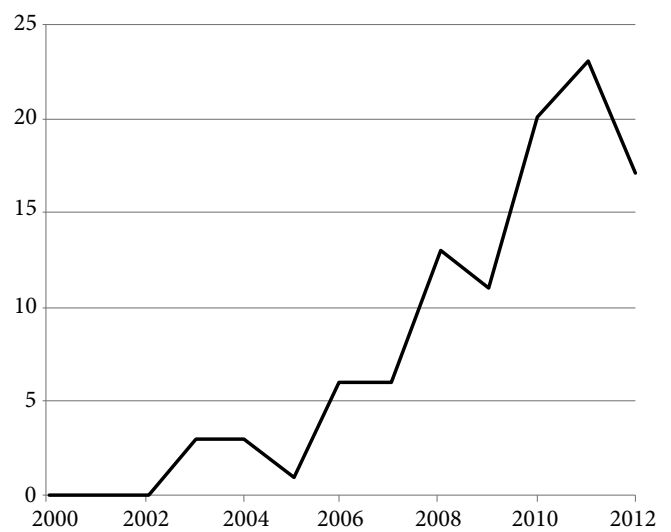

Fuente: elaboración propia con los datos mencionados. 
Es posible ver que los países con mayor participación en la escritura de los artículos son Estados Unidos, España y el Reino Unido (Fig. 10).

Figura 10. Países de los autores y coautores, además de México

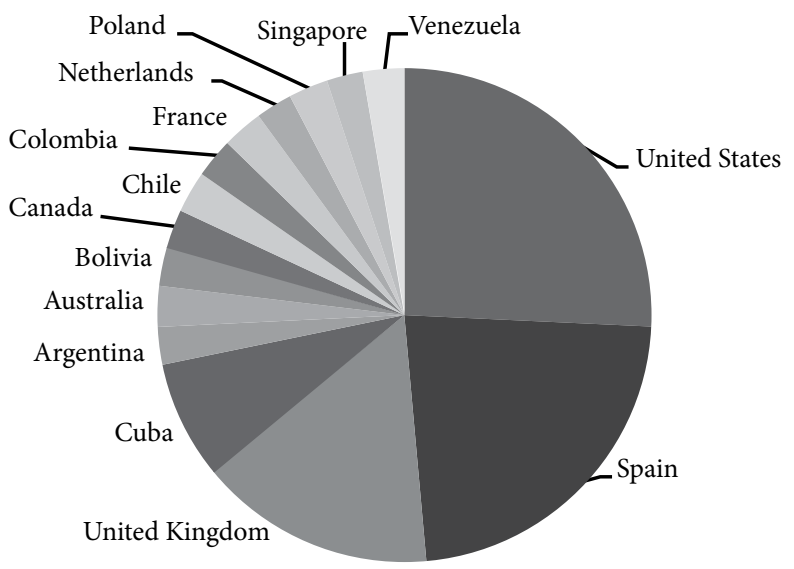

Fuente: elaboración propia con los datos mencionados.

En cuanto a la colaboración interinstitu- de Barcelona y la Universidad Autónoma cional, destacan la Universidad Autónoma Metropolitana (Fig. 11).

Figura 11. Institución de adscripción de autores y coautores (sólo las nueve primeras)

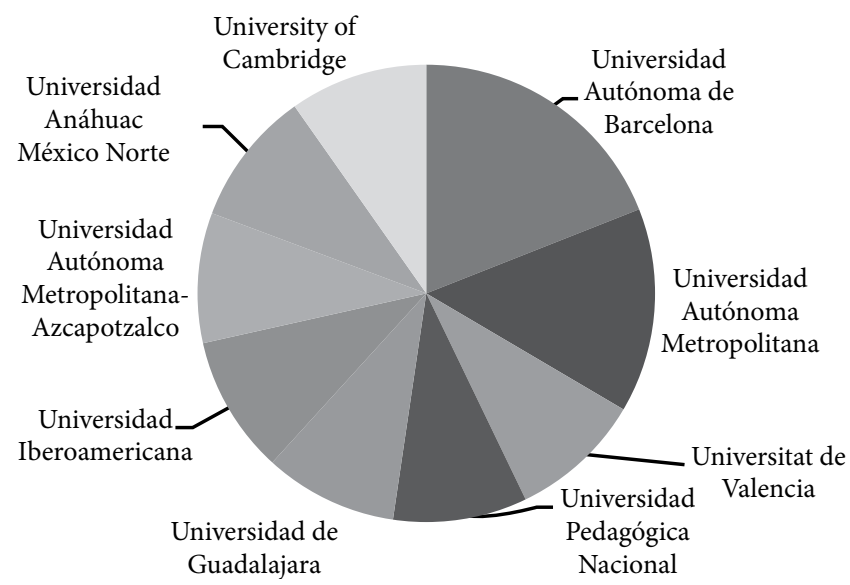

Fuente: elaboración propia con los datos mencionados.

Por último, y en virtud de que la base de datos lo permite, la Fig. 12 indica las áreas que tocan los artículos publicados. Destacan, de manera congruente con lo que ya se ha visto, Química y Medicina. 
Figura 12. Área de los artículos publicados en Scopus

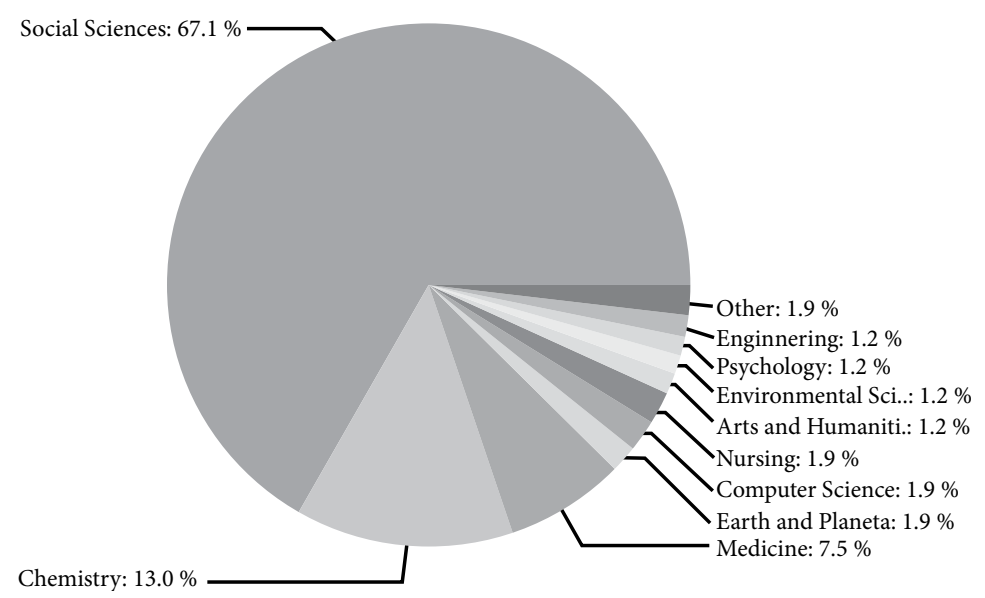

Fuente: elaboración propia con los datos mencionados.

\section{Discusión}

Como puede verse, el número de revistas de investigación educativa catalogadas por el CONACyT es bajo, y de ellas, sólo tres aparecen en los índices líderes. El índice JCR contiene pocas revistas en idioma español, casi todas provenientes de España. Publicar en español puede resultar más sencillo para nuestros investigadores, pero limita las posibilidades de ingresar a los índices líderes y reduce la visibilidad de los artículos publicados. También puede observarse que las revistas indizadas en español tienen factores de impacto más bien bajos.

Las cinco revistas líderes en el JCR se abocan a temas como administración del aprendizaje y la educación; aprendizaje e instrucción; investigación educativa e investigación acerca de la lectura. Las revistas mexicanas se dirigen a la investigación educativa en general, a la matemática educativa y a la educación superior. Puede decirse que los dos tipos de revistas tienen enfoques semejantes

Se observó que las normas para los autores que deseen colaborar son muy similares en ambos tipos de revistas (nacionales e internacionales), con la excepción de que las revistas indexadas en JCR suelen especificar que los artículos no sólo deben ser escritos en inglés, sino en "buen" inglés. Algunas inclusive detallan que debe usarse ya sea inglés americano o inglés británico, sin mezclar ambos. Puede notarse que el formato IMRyD, de amplia utilización en las ciencias fácticas, no es un requisito fundamental para los artículos de investigación dentro de la investigación educativa. Esto pudiera brindar un poco más de libertad a los autores potenciales.

En cuanto a la producción de artículos de investigación educativa dentro de revistas catalogadas por los índices que son, hoy por hoy, los líderes en el mundo académico, los datos revelan que es baja: 18 artículos en CCC y 107 en Scopus. Llama la atención que en el índice JCR la mayoría de los artículos registrados por autores adscritos a la UNAM se refiere a la educación en Química y Medicina. Los autores con más artículos pertenecen también a las facultades de Química y Medicina, aunque los datos son muy pocos como para concluir algo acerca de ellos. La colaboración con países e instituciones se encuentra atomizada $y$, por lo mismo, tampoco se detectan patrones o tendencias.

Desde un discurso oficial y con una mirada poco analítica, los resultados podrían interpretarse como un indicador de que la productividad académica en la UNAM y en México, en el ámbito de la investigación educativa, es baja en cantidad y calidad, puesto 
que no figura en las revistas con mayores índices de impacto mundial. Sin embargo, consideramos que es necesario profundizar en este problema con una perspectiva reflexiva y crítica.

En primera instancia, la producción académica reducida podría simplemente confirmar el hecho de que, según Ramírez y Weiss (2004), el número de investigadores educativos es relativamente bajo en nuestro país $y$, por ende, en la UNAM. Si se toma a los investigadores del área de Pedagogía como un acercamiento a los investigadores educativos (Fig. 3) en México, efectivamente es así. Sin embargo, es importante recalcar que existen investigadores educativos en otras áreas del conocimiento. Los datos de este trabajo precisamente revelan que se está publicando investigación educativa en áreas como Química, Medicina y Matemáticas.

Resulta claro también que los investigadores educativos parecen preferir las publicaciones en español, o que tal vez tienen dificultades para traducir de manera adecuada sus artículos al idioma inglés, como lo solicita la mayor parte de las revistas indizadas en JCR o Scopus. Sin embargo, la producción de artículos de investigación en otras áreas como la Física o la Química es mucho mayor en la UNAM, aun cuando enfrenta el mismo problema del idioma. Cabe preguntarse por qué en estas áreas se produce más. Sin embargo, también debe enmarcarse esta situación en el contexto mundial. En todo el mundo se producen, en general, más artículos de investigación en áreas como Física, Química y Medicina. Esto pudiera responder a criterios de tipo económico; es decir, estas investigaciones producen patentes, medicamentos, dispositivos, etcétera, que tienen un fuerte valor económico hoy en día. Esto pudiera ser parte de la razón por la cual más personas se dirigen -y más fondos se destinan - a la investigación en estas áreas.

También es factible que el número de registros de adscripción a la UNAM en las publicaciones referidas sea bajo porque existen errores y variantes en la forma de capturar este dato. Algunos académicos laboran en varias instituciones a la vez y registran aquella en la que estaban más involucrados al hacer la investigación, o aquella que otorgó fondos para el proyecto. Esto pudiera dejar fuera artículos con datos registrados en forma equivocada o con autores adscritos a la UNAM que indicaron otra adscripción en su artículo. Sin embargo, estos problemas no deberían ser muy significativos.

Por otro lado, las revistas indizadas en JCR y Scopus tienen niveles de exigencia altos. Los trabajos que se propongan deben seguir desde lineamientos de formato muy específicos, hasta contenidos de contribución significativa al conocimiento, expresados con lenguaje claro y preciso. Las referencias que se citen deben ser también de excelente calidad, y mostrar un panorama completo y crítico del estado del arte. Además, la mayor parte de las revistas solicitan que los manuscritos se envíen en un inglés correcto. El procedimiento para enviar los artículos es a través de plataformas tecnológicas que tienen cierto nivel de complejidad y que también suelen estar en inglés, al igual que los comunicados del editor y los árbitros. Algunos investigadores educativos pudieran sentirse agobiados ante estas exigencias.

Los autores de este trabajo han tenido la oportunidad de participar como árbitros, tanto de revistas de investigación educativa de alto impacto, como de libros y otras publicaciones. En general hemos encontrado que este trabajo es muy enriquecedor y se convierte en un camino didáctico donde se comparte, corrige y perfecciona el conocimiento entre pares. Sin embargo, tal vez sea necesario apoyar la formación de los investigadores y animarlos a participar en este ámbito.

Otro aspecto relevante es que la mayoría de la investigación educativa mexicana se enfoca en problemas locales o domésticos. Esto por sí mismo es correcto, ya que en los países 
desarrollados también se abocan a problemas domésticos, pero suelen enmarcarlos en discusiones disciplinarias o teóricas mucho más amplias. Sus apartados teóricos ubican el problema dentro de la literatura y la discusión mundiales. Esto sugiere que, probablemente, sea necesario desarrollar la significación global de los problemas locales a los que se circunscribe la indagación actual, para aspirar a publicar en revistas internacionales.

Ahora, a partir de la revisión que hemos hecho en este trabajo, parecería que estos índices, series periódicas, citas y factores de impacto son las únicas formas válidas y objetivas de juzgar el trabajo académico, en general y particularmente en la investigación educativa. Sin embargo, la educación, más allá de las publicaciones, es un campo de práctica (García Cabrero et al., 2008).

Muchos de los trabajos académicos que hacen los investigadores educativos se traducen en productos y servicios cuya utilidad principal no es generar una publicación de alto impacto, sino contribuir a reformular políticas educativas; reformar o actualizar planes y programas de estudio; desarrollar materiales didácticos, o mejorar la práctica docente en el aula. También ocurre que algunos de estos trabajos se publican en medios y formatos que, si bien son distintos de las series periódicas, resultan útiles tanto para mejorar programas educativos como para apoyar la formación docente. Uno de los autores de este artículo ha publicado artículos en periódicos de circulación nacional con el fin de llegar así a un público más amplio, pero con una estructura formal y rigurosa, que incluye bibliografía y citación de resultados de investigación. Estos artículos han sido reproducidos por diversos docentes y autoridades educativas en México y otros países, quienes señalan la utilidad de estas publicaciones para sus tareas de formación de maestros, diseños curriculares, y como material de lectura para cursos de licenciatura y posgrado. Otro de los autores ha cristalizado resultados de investigación de gabinete y documental, también de tesis doctoral, en la constitución de ambientes virtuales de aprendizaje que dan servicio a más de seis mil estudiantes, en boletines de difusión tecnológica, en libros de divulgación y en múltiples cursos de formación docente.

Estos ejemplos sugieren la necesidad de repensar las formas en que se evalúan, valoran y legitiman los resultados de la investigación educativa. Si se continúa privilegiando únicamente a las publicaciones indizadas dentro de series periódicas con factores de impacto elevados, los casos que hemos descrito seguirán siendo negados, devaluados e ignorados.

Lo anterior también se relaciona con el hecho de que en México existen dos grandes categorías de investigadores educativos: por un lado, los que pertenecen a institutos y centros de investigación, cuyo trabajo primordial es precisamente investigar, y que cuentan con las mejores condiciones para ello; ${ }^{4}$ por el otro, los profesores cuyo trabajo sustancial es la atención a grupos, casi siempre de licenciatura, además de la revisión de planes y programas de estudio, asistencia a reuniones y comisiones, tutorías, asesorías, aplicación de exámenes, entre muchas otras. Y todavía en este segundo grupo podría hacerse diferencia entre los profesores de carrera y los de asignatura. Estos últimos conforman 70 por ciento del personal académico de la UNAM (UNAM, 2011) y se hacen cargo de la mayor parte de la docencia que atiende a los 300 mil alumnos de esta institución. Estos profesores tienen una remuneración simbólica: 299.56 pesos la hora-semana-mes, o 74.89 pesos la hora, para profesor de asignatura A (AAPAUNAM, 2013) y es difícil esperar que produzcan tanto

4 Un profesor o investigador titular C de tiempo completo en la UNAM tiene un sueldo mensual de 24,964.84 pesos (AAPAUNAM, 2013) al que puede agregarse un porcentaje de entre 85 y 115 por ciento si se pertenece al programa de estímulos de mayor nivel, más la antigüedad académica y otras prestaciones. 
estudiantes graduados como publicaciones de alta calidad. ${ }^{5}$

Los programas de estímulo y reconocimiento evalúan con idénticas normas a los dos grandes grupos que hemos descrito y valoran, ante todo, la producción de publicaciones en revistas indizadas y las citas en las mismas (Rueda Beltrán et al., 2010). Por poner un ejemplo, se otorga más valor a publicar un artículo de 20 cuartillas en una revista internacional, que a sacar adelante diez generaciones de alumnos de licenciatura. En la Universidad Autónoma Metropolitana, el Tabulador de Ingreso y Promoción del Personal Académico otorga alrededor de 200 puntos por impartir una clase a alumnos de licenciatura, mientras que publicar un artículo de investigación se valora hasta con $3 \mathrm{mil}$ 300 puntos, según la "categoría de la revista" (UAM, 2003). Los artículos de alto impacto son el "nuevo becerro de oro" (García, 2012) de los académicos actuales y, a decir de T. Stewart (2006): "son las publicaciones, y no la excelencia en la enseñanza, las que brindan las recompensas de permanencia, promoción, seguridad y prestigio".

Ahora bien, publicar artículos de investigación en revistas con alto impacto no es sólo cuestión de capacidad y calidad de un investigador que trabaje de manera individual. Igual que en los deportes de equipo, jugar en las grandes ligas exige ser parte de una red académica internacional. Participar en esta red puede ser relativamente accesible cuando se pertenece al primer grupo de investigadores educativos, quienes tienen la infraestructura al servicio de esta finalidad. Los centros o institutos convocan y realizan eventos con invitados de categoría mundial, que brindan espacios propicios para crear diálogos y coloquios personales. Los investigadores pueden viajar y conectarse con sus pares. Cuentan con ayudantes para labores que requieren mucho tiempo y para búsquedas documentales. Se les apoya también en su trabajo frente a grupo, cuando lo tienen. Sin duda, es razonable la exigencia de producir investigaciones de alto impacto para este grupo.

Además, la investigación implica contar con tiempo de calidad para realizar labores de observación, selección, recuperación y análisis crítico de muchos documentos; recogida y procesamiento de datos; interpretación racional a la luz de una o varias teorías; comparación con resultados de otros investigadores; escritura de reportes, lectura cuidadosa de los dictámenes de los árbitros, entre muchas otras. ¿Es compatible todo esto con el tiempo necesario para atender de manera adecuada la función docente? Y no sólo se trata de contar con espacios temporales; éstos deben ser de calidad y tener una longitud mínima. La ventana de creación intelectual implica que el investigador pueda trabajar y reflexionar sobre un tema por un tiempo prolongado. Es muy difícil brincar de la lectura de un artículo de revisión a la atención de un alumno con dudas, para luego continuar otro poco y correr al salón de clase; proseguir la lectura y dejarla para calificar tareas y corregir exámenes... y así sucesivamente, día tras día.

Consideramos que continuar con esta misma exigencia hacia los profesores de carrera y asignatura, a través de los programas de estímulo y reconocimiento académicos, sobrepasa las capacidades y condiciones que la propia institución otorga a estos docentes. Esta exigencia genera una sensación de falta de reconocimiento y frustración en muchos de los docentes, y se basa en la suposición de que quienes son capaces de contribuir a la creación de conocimiento, serán también mejores profesores (Rueda Beltrán et al., 2010). Además, la docencia y la investigación son trabajos distintos, que requieren formaciones distintas, condiciones distintas e inclusive, son el resultado de vocaciones académicas distintas. La evaluación de los profesores, más

5 Es decir, mientras un investigador de tiempo completo puede llegar a tener un sueldo mensual de cerca de $100 \mathrm{mil}$ pesos, un profesor de asignatura, con una materia, tiene un sueldo de 1 mil 200 pesos mensuales. 
orientada a la investigación que a la docencia, ha ocasionado que hoy en día se perciban las clases como una carga (Stewart, 2006) -de hecho, así se le llama: "carga docente"- que impide o dificulta el trabajo más reconocido de investigar y publicar.

Coincidimos con Rueda et al. (2010), en cuanto a que no parece haber evidencia de que los programas de estímulos y reconocimientos mejoren la docencia, pero además, según nuestros datos, tampoco parecen haber incidido de manera positiva en la producción de publicaciones de investigación en revistas de alto impacto, en el ámbito de la investigación educativa. Tal vez los requerimientos de los programas de estímulos y reconocimientos basados en el rendimiento no son apropiados para motivar este tipo de producciones $\mathrm{o}$, inclusive, pudieran estar actuando de forma contraproducente. Estos programas han sido criticados prácticamente desde su inicio (Díaz-Barriga, 1996) y a lo largo de su funcionamiento (Heras, 2005; Sánchez Dromundo, 2005). Los cuestionamientos se refieren, entre otras cosas, a que estos sistemas de pago por mérito prácticamente excluyen y devalúan la labor docente, al mismo tiempo que producen frustración, estrés y problemas de salud (Martínez, 2012; Urquidi, 2010), promueven la competencia entre los profesores, y borran la distinción entre las funciones de docencia e investigación, al crear la figura de docente-investigador.

Pero además de estos problemas - que ameritan contar con su propia agenda de discusión-, los autores mencionados brindan evidencias que sugieren que estos programas tienden a deteriorar la calidad de las publicaciones alrededor de la investigación, en beneficio de la cantidad y la proliferación de refritos 6 e investigaciones de poca monta. Para cumplir con estándares que son más de cantidad que de calidad, los investigadores invertimos nuestro tiempo en escritos que sean breves y poco profundos, que nos permitan obtener resultados rápidos que puedan reutilizarse en diversos foros y medios, para con ello conseguir los puntos necesarios para ingresar o permanecer en los programas. Tal vez estamos buscando publicar en revistas con menos exigencias, o tal vez las revistas no indizadas en JCR y Scopus son las más adecuadas para nuestro objeto de estudio, o tal vez muchos de nosotros simplemente no estamos publicando artículos de investigación.

La existencia de estos programas de estímulos y reconocimiento mantiene en pie la necesidad de debatir acerca de cómo definir la línea entre el mayor o menor valor del trabajo académico en el ámbito educativo. Hoy por hoy ofrece mayor retribución, prestigio y remuneración económica, publicar artículos que contribuir al mejoramiento de la labor docente. Se busca el reconocimiento de un grupo diminuto de pares académicos y se deja de lado el reconocimiento a productos y beneficios reales, palpables, de la labor educativa.

Por otro lado, habría que determinar cuáles son las urgencias en el sistema educativo mexicano y, en general, en los países en vías de desarrollo. Sin duda, los países desarrollados producen grandes cantidades de artículos de investigación, pero la mayoría de ellos se enfocan en examinar problemas prácticos. La diferencia es que insertan el planteamiento $y$ la solución de estos problemas en discusiones teóricas de relevancia global. Parecería deseable que nosotros, como investigadores educativos, participáramos también en estas discusiones.

En todo caso, parece sustancial revalorar la función docente que tiene una importancia significativa para nuestro país, puesto que está directamente relacionada con la formación de los jóvenes. Por otro lado, si se desea que México incremente su participación

6 De acuerdo con la Real Academia Española, refrito significa, en su segunda acepción: "Cosa rehecha o recompuesta, especialmente refundición de una obra dramática o de otro escrito". 
mundial en las publicaciones extranjeras con calificaciones altas, será indispensable revisar las políticas relacionadas con ciencia y tecnología. Debe analizarse cuál es la conveniencia de mejorar los índices de desarrollo global asociados con este factor y, eventualmente, cuáles son las mejores vías para conseguir estas metas.

Dentro de las limitaciones de esta investigación podemos mencionar que las búsquedas que realizamos son necesariamente incompletas, como se mencionó en la sección de metodología. Asimismo, consideramos que nuestra posición ante el objeto de estudio es

\section{REFERENCIAS}

Asociación Autónoma del Personal Académico de la Universidad Nacional Autónoma de México (AAPAUNAM) (2013), Contrato colectivo de trabajo del personal académico 2013-2015, México, UNAM.

Consejo Nacional de Ciencia y Tecnología (CONAСуТ) (2012), Índice de revistas mexicanas de investigación científica y tecnológica en México, en: http://www.redalyc.org/conacyt/ busquedas/IndexacionesPorArea.jsp?Cmb Rev=4 (consulta: 10 de septiembre de 2012).

Consejo Nacional de Ciencia y Tecnología (CONAСуT) (2013), Estadística básica 2012, en: http:// geo.virtual.vps-host.net:8080/SIICYT/estadisticas.do? method $=$ iniCiencia\&anio $=2012$ (consulta: 3 de abril de 2013).

DíAz-Barriga, Ángel (1996), "Los programas de evaluación (estímulos al rendimiento académico) en la comunidad de investigadores. Un estudio en la UNAM", Revista Mexicana de Investigación Educativa, vol. 1, núm. 2, pp. 408-423.

ElSevier (2013), Scopus, en: http://www.scopus. com/home.url (consulta: 12 de septiembre de 2012).

García Cabrero, Benilde, Javier Loredo Enríquez y Guadalupe Carranza Peña (2008), "Análisis de la práctica educativa de los docentes: pensamiento, interacción y reflexión", Revista Electrónica de Investigación Educativa, vol. 10, núm. especial, pp. 1-15.

García R., Enrique (2012), "Reflexionando en torno a la investigación educativa: una mirada crítica desde la auto etnografía de un docente", Qualitative Research in Education, vol. 1, núm. 1, pp. 58-79. necesariamente no neutral, puesto que forma parte de nuestras metas y actividades cotidianas, lo cual es inseparable de nuestra afectividad y percepción. Sin embargo, el problema es complejo y exige una mirada cualitativa y humana que complemente el análisis de los datos estadísticos, de ahí que hemos expresado nuestra opinión, razonada y fundamentada.

Será deseable continuar el estudio de este problema incorporando de manera amplia la perspectiva de todos sus actores: investigadores educativos, docentes, evaluadores, y responsables de políticas educativas y económicas.

GARfield, Eugene (2006), “The History and Meaning of the Journal Impact Factor", Journal of the American Medical Association (JAMA), núm. 293, pp. 90-93.

Heras, Leticia G. (2005), "La política de educación superior en México: los programas de estímulos a profesores e investigadores”, Revista Venezolana de Educación (Educere), vol. 9, núm. 29, pp. 207-215.

Martínez Alcántara, Susana, Ignacio Méndez Ramírez y Chiharu Murata (2011), "Becas y estímulos y sus consecuencias sobre el trabajo y la salud de docentes universitarios", Reencuentro, núm. 61, pp. 56-70.

Merton, Robert K. (1968), "The Matthew Effect in Science", Science, vol. 159, núm. 3810, pp. 56-63.

Ramírez, Rosalba y Eduardo Weiss (2004), "Los investigadores educativos en México: una aproximación", Revista Mexicana de Investigación Educativa, vol. 9, núm. 21, pp. 501-513.

Rueda Beltrán, Mario, Edna Luna Serrano, Benilde García Cabrero y Javier Loredo Enríquez (2010), "La evaluación de la docencia en las universidades públicas mexicanas: un diagnóstico para su comprensión y mejora", Revista Iberoamericana de Evaluación Educati$v a$, vol. 3, núm. le, pp. 77-92.

Sánchez Dromundo, Rosalba A. (2005), "El impacto de los programas de estímulos de los académicos del posgrado", en Raquel Glazman Nowalski (ed.), Las caras de la evaluación educativa, México, UNAM, pp. 103-129.

SEgLen, Per O. (1997), "Why the Impact Factor of Journals should not be Used for Evaluating Research", BMJ, vol. 314, núm. 7079, pp. 497-502. 
STEWART, Timothy(2006), “Teacher-ResearcherCollaboration or Teachers' Research?”, TESOL Quarterly, vol. 40, núm. 2, pp. 421-430.

Testa, James (2012), The Thomson Reuters Journal Selection Process, Thomson Reuters, en: http:// thomsonreuters.com/products_services/science/free/essays/journal_selection_process/ (consulta: 12 de septiembre de 2012).

The World Bank (2012), Scientific and Technical Journal Articles, en: http://data.worldbank.org/ indicator/IP.JRN.ARTC.SC (consulta: 12 de septiembre de 2012).

Thomson Reuters (2011), Journal Citation Reports: Social sciences edition, en: http://isiknowledge.com (consulta: 7 de septiembre de 2012).

Thomson Reuters (2012), Journal Citation Reports, en: http://wokinfo.com/products_tools/ analytical/jcr/ (consulta: 10 de septiembre de 2012).

Thomson Reuters (2013), Web of Knowledge, en: http://isiknowledge.com (consulta: 30 de mayo de 2013).

Universidad Nacional Autónoma de México (2011), Agenda Estadística, en: http://www.planeacion.unam.mx/Agenda/2011/disco/\# (consulta: 5 de febrero de 2013).
Universidad Nacional Autónoma de México (UNAM)-Dirección General de Asuntos del Personal Académico (DGAPA) (1996), Lineamientos y requisitos generales para la evaluación de profesores e investigadores, en: http:// dgapa.unam.mx/Reglamentos/LinProfInvpride.pdf (consulta: 12 de septiembre de 2012).

University of Pennsilvanya (2011), Eugene Garfield, en: http://www.garfield.library.upenn.edu/ (consulta: 10 de septiembre de 2012).

URQUIDI Treviño, Laura Elena y José Raúl Rodríguez Jiménez (2010), "Estrés en profesorado universitario mexicano", Actualidades Investigativas en Educación, vol. 10, núm. 2, pp. 1-21.

WeIss, Eduardo y Norma G. Gutiérrez (2003), “Comunicación de la investigación educativa”, en Eduardo Weiss (ed.), El campo de la investigación educativa, 1993-2001, México, Consejo Mexicano de Investigación Educativa (COMIE), pp. 172-192.

Ziman, John Michael (1968), Public Knowledge: An essay concerning the social dimension of science, Londres, Cambridge University Press. 